\title{
BER Analysis Using Beat Probability Method of 3D Optical CDMA Networks with Double Balanced Detection
}

\author{
Chih-Ta Yen ${ }^{1}$ and Chih-Ming Chen ${ }^{2}$ \\ ${ }^{1}$ Department of Electrical Engineering, National Formosa University, Yunlin County 632, Taiwan \\ ${ }^{2}$ Department of Electrical Engineering, Institute of Computer and Communication Engineering, National Cheng Kung University, \\ Tainan 701, Taiwan \\ Correspondence should be addressed to Chih-Ta Yen; chihtayen@gmail.com
}

Received 16 September 2014; Accepted 7 January 2015

Academic Editor: Mo Li

Copyright (C) 2015 C.-T. Yen and C.-M. Chen. This is an open access article distributed under the Creative Commons Attribution License, which permits unrestricted use, distribution, and reproduction in any medium, provided the original work is properly cited.

This study proposes novel three-dimensional (3D) matrices of wavelength/time/spatial code for code-division multiple-access (OCDMA) networks, with a double balanced detection mechanism. We construct 3D carrier-hopping prime/modified prime (CHP/MP) codes by extending a two-dimensional (2D) CHP code integrated with a one-dimensional (1D) MP code. The corresponding coder/decoder pairs were based on fiber Bragg gratings (FBGs) and tunable optical delay lines integrated with splitters/combiners. System performance was enhanced by the low cross correlation properties of the 3D code designed to avoid the beat noise phenomenon. The CHP/MP code cardinality increased significantly compared to the CHP code under the same bit error rate (BER). The results indicate that the $3 \mathrm{D}$ code method can enhance system performance because both the beating terms and multiple-access interference (MAI) were reduced by the double balanced detection mechanism. Additionally, the optical component can also be relaxed for high transmission scenery.

\section{Introduction}

Optical code-division multiple-access (OCDMA) systems are considered suitable for local-area networks because they allow multiple users to access the network asynchronously and simultaneously with high transmission security $[1,2]$ especially when the code dimensional becomes large. Several methods have been proposed to achieve passive OCDMA. Most types of OCDMA systems use optical delay lines and optical orthogonal codes for time domain coding [1]. However, some OCDMA systems employ spectral coding through diffraction gratings, confocal lenses, and phase/amplitude masks [3]. Under these OCDMA schemes, optical systems require coherent and ultrashort pulses to produce transformlimited pulses, increasing the cost and complexity of the system.

In earlier OCDMA systems, one-dimensional (1D) codes such as optical orthogonal codes (OOCs) [1] were used. However, the code length must be sufficient to support additional users because of the unipolar characteristic of optical signals. The disadvantage of $1 \mathrm{D}$ coding systems is that the code length increases rapidly when the number of active users increases. To overcome the limitations of $1 \mathrm{D}$ codes, multidimensional coding methods, using two-dimensional (2D) codes and three-dimensional (3D) codes, were proposed [4-7].

These researches present new 3D matrices and an associated decoding scheme for a wavelength/time/spatial OCDMA system. We constructed 3D carrier-hopping prime/modified prime (CHP/MP) codes by extending a 2D CHP code integrated with a $1 \mathrm{D}$ MP code. In the 3D code matrices, a CHP code is used for wavelength/time coding and a MP code is used for spatial coding. System performance was enhanced by the low cross correlation properties of the 3D code designed to avoid the beat noise phenomenon. At the receiver, multiuser interference can also be suppressed efficiently using the double balanced detection scheme. In general, the proposed 3D security performance is better than $2 \mathrm{D}$ and $1 \mathrm{D}$ OCDMA networks. The research group of Shake [8] suggested that there are three issues 
for enhancing the network security to protect the network from attack by unauthorized users. In the first, the approach involved increasing the code complexity, for example, more dimensions or more complexity code sets, while the second involved reducing the subscriber's transceiver power. A third issue is for each transmitter to change its code pattern on a frequent basis. One of the advantages in our proposed $3 \mathrm{D}$ OCDMA network can enhance security when the network is under attack from the eavesdroppers. The remainder of this paper is organized as follows. Section 2 provides an overview of the $3 \mathrm{D}$ code construction and its properties. Section 3 provides an illustration of the system configuration. Section 4 evaluates the system performance and discusses the dominated noise effects. Finally, Section 5 presents some brief conclusions.

\section{Code Construction and Code Properties}

The wavelength/time/spatial 3D code can be constructed following the steps outlined below. In this study, we first constructed CHP code used for the $2 \mathrm{D}$ wavelength/time code. Then, we used the MP code to spatially code the 3D code matrices. Finally, we combined the CHP codes with MP codes to generate $3 \mathrm{D}$ code matrices labeled $3 \mathrm{D} \mathrm{CHP} / \mathrm{MP}$ codes in this study.

The code construction process is as follows.

Step 1. With a positive integer $t$ and two prime numbers $p_{1}$ and $p_{2}$, the $p_{1} \geq p_{2} \geq t$, code matrices $\mathbf{A}_{\alpha, \beta}$ can be expressed as

$$
\mathbf{A}_{\alpha, \beta}=\left[\begin{array}{ccc}
a_{0,0} & \cdots & a_{0, p_{1} p_{2}-1} \\
\vdots & \ddots & \vdots \\
a_{t-1,0} & \cdots & a_{t-1, p_{1} p_{2}-1}
\end{array}\right]=\left[a_{x, y}\right]_{t \times p_{1} p_{2}}
$$

where $\alpha=\left\{0,1, \ldots, p_{1}-1\right\}$ and $\beta=\left\{0,1, \ldots, p_{2}-1\right\}$. The element $a_{x, y}$ is an element of $\mathbf{A}_{\alpha, \beta}$ and can be expressed using

$$
a_{x, y}=\left\{\begin{array}{l}
1, \quad \text { if } x=0, \quad y=0 \\
x=1, \quad y=\alpha=\beta p_{1} \\
x=2, \quad y=2 \odot_{p_{1}} \alpha=\left(2 \odot_{p_{2}} \beta\right) p_{1} \\
\vdots \\
x=t-1, \\
y=(t-1) \odot_{p_{1}} \alpha=\left((t-1) \odot_{p_{2}} \beta\right) p_{1} \\
0, \quad \text { otherwise, }
\end{array}\right.
$$

where $\odot_{N}$ denotes modulo- $N$ multiplication. For example, if we set $t=p_{1}=p_{2}=3$, the CHP code has nine matrices. Table 1 shows this type of CHP code family.

Step 2. The construction of a modified prime code begins with the Galois field GF( $p)$ of a prime number $p$. A modified prime sequence $y_{\mu, \nu}(z)$ is constructed with elements of $\mathrm{GF}(p)$ over an odd prime using the following expression:

$$
y_{\mu, v}= \begin{cases}(\mu+v z) \odot p, & z=0,1, \ldots, p-1 \\ v, & z=p,\end{cases}
$$

where $\mu \in\{0,1, \ldots, p-1\}$ and $\nu \in\{0,1, \ldots, p-1\}$.

Therefore, we can generate different $p^{2}$ sequences by changing parameters $\mu$ and $\nu$. These $p^{2}$ sequences form a code family.

To construct the modified prime code each modified prime sequence is mapped into a binary $(0,1)$ modified prime code sequence $\mathbf{B}_{\gamma}=b \gamma(0), b \gamma(1), \ldots, b \gamma(i), \ldots, b \gamma\left(p^{2}+p-1\right)$ of length $p^{2}+p$ according to

$$
b_{\gamma}(i)= \begin{cases}1, & \text { if } i=z p+y_{\mu, v}(z) \\ 0, & \text { otherwise }\end{cases}
$$

where $i=0,1, \ldots, p^{2}+p-1, \gamma=0,1, \ldots, p^{2}-1$, and $\gamma=$ $p \times \mu+\nu$.

Step 3. Generate code matrices $\mathbf{C}_{\alpha, \beta, \gamma}$ using the following mathematical expression:

$$
\mathbf{C}_{\alpha, \beta, \gamma}=\left|\begin{array}{c}
{\left[b_{\gamma}(0) A_{\alpha, \beta}\right]} \\
{\left[b_{\gamma}(1) A_{\alpha, \beta}\right]} \\
\vdots \\
{\left[b_{\gamma}\left(p^{2}+p-1\right) A_{\alpha, \beta}\right]}
\end{array}\right|=\left[c_{x, y, z}\right]_{t \times p_{1} p_{2} \times p^{2}},
$$

where $\alpha=\left\{0,1, \ldots, p_{1}-1\right\}, \beta=\left\{0,1, \ldots, p_{2}-1\right\}$, and $\gamma=$ $\left\{0,1, \ldots, p^{2}-1\right\} . c_{x, y, z}$ is an element of $\mathbf{C}_{\alpha, \beta, \gamma}$. When element $c_{x, y, z}=1$, the optical pulse of wavelength $x$ exists at time chip $y$ in spatial channel $z$.

Then, we define the $3 \mathrm{D}$ code using a mathematical expression.

Definition 1. A $\left(t \times p_{1} p_{2} \times\left(p^{2}+p\right), w, \lambda_{a}, \lambda_{c}\right) 3 \mathrm{D}$ code $\mathbf{C}$ is a collection of binary $(0,1) t \times p_{1} p_{2} \times\left(p^{2}+p\right)$ matrices $\mathbf{C}_{\alpha, \beta, \gamma}$, each at hamming weight $w$, satisfying the following constraints.

Autocorrelation. For all 3D codewords $\mathbf{S}, \mathbf{R} \in \mathbf{C}$

$$
\begin{aligned}
\sum_{z=0}^{p^{2}+p-1} \sum_{x=0}^{t-1} \sum_{y=0}^{p_{1} p_{2}-1} s_{x, y, z} s_{x, y \oplus \tau, z} & =w, \quad \text { for } \tau=0 \\
& \leq \lambda_{a} \text { for } 1 \leq \tau \leq p_{1} p_{2}-1 .
\end{aligned}
$$

Cross Correlation. For all 3D codewords $\mathbf{S}, \mathbf{R} \in \mathbf{C}$

$$
\sum_{z=0}^{p^{2}+p-1} \sum_{x=0}^{t-1} \sum_{y=0}^{p_{1} p_{2}-1} s_{x, y, z} s_{x, y \oplus \tau, z} \leq \lambda_{c}, \quad \text { for } 0 \leq \tau \leq p_{1} p_{2}-1
$$


TABle 1: CHP codes for $T=P_{1}=P_{2}=3$.

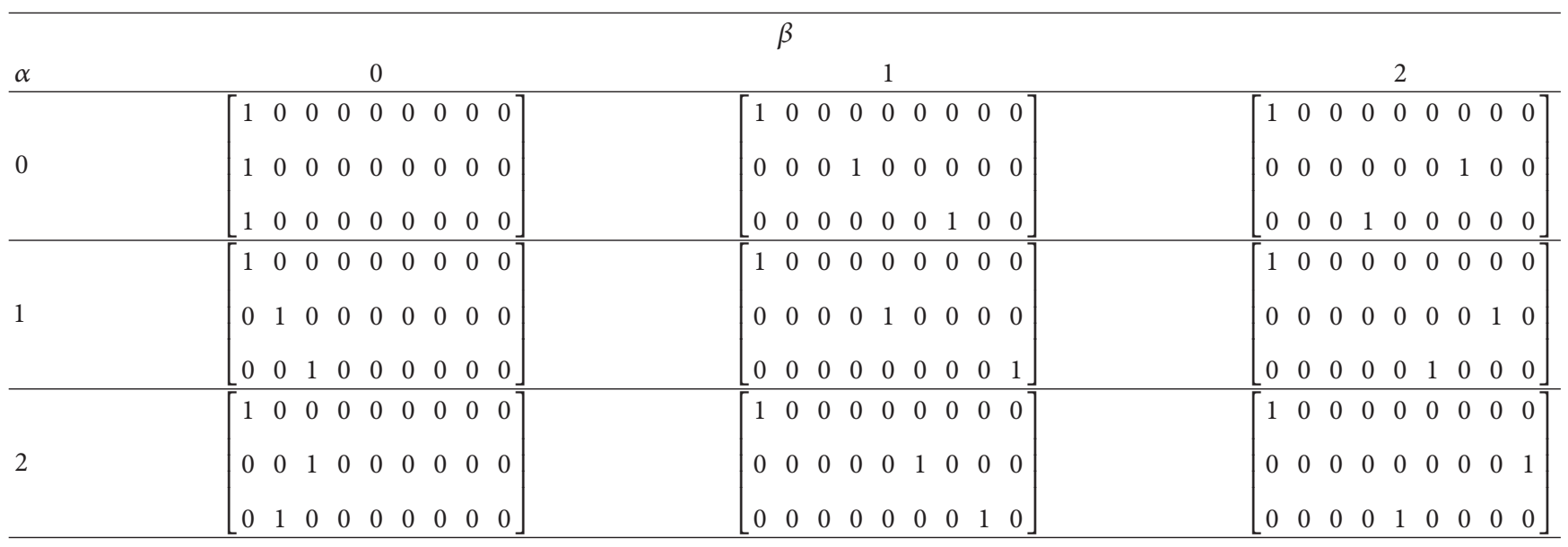

TABLE 2: Correlation properties of CHP/MP codes.

\begin{tabular}{lcc}
\hline & Cross correlation & Autocorrelation \\
\hline Case 1 & $\lambda_{c}=t$ & $\lambda_{\alpha}=0$ \\
Case 2 & $\lambda_{c}=p+1$ & $\lambda_{\alpha}=0$ \\
Case 3 & $\lambda_{c}=1$ & $\lambda_{\alpha}=0$ \\
\hline
\end{tabular}

where $s_{x, y, z}$ and $r_{x, y, z}$ are the elements of matrices $\mathbf{S}_{\alpha, \beta, \gamma}$ and $\mathbf{R}_{\alpha, \beta, \gamma}$, respectively, and a nonnegative integer $\lambda_{c}$ represents cross correlation.

The proposed code properties can be divided into three types: (1) two distinct codes with the same wavelength/time coding scheme; that is, both codes have the same CHP code codeword but different spatial coding schemes. In other words, they have the same modified prime code codeword; (2) two distinct codes with different wavelength/time coding schemes but the same spatial coding schemes; (3) two distinct codes with different wavelength/time coding schemes and different spatial coding schemes. The cross correlation and autocorrelation properties of 3D CHP/MP codes are shown in Table 2. The values in Table 2 show that the worst cross correlation condition would be obtained by comparing Case 1 with Case 2 . However, these results have been omitted from this paper; interested readers can find them in [8].

\section{System Configuration of 3D OCDMA Network}

Figure 1 shows a block diagram of the proposed 3D OCDMA network. The coding scheme uses $t \times p_{1} p_{2} \times\left(p^{2}+p\right)$ matrices, where $t$ is the number of available wavelengths, $p_{1} p_{2}$ is the number of time slots, and $p^{2}+p$ is the number of spatial channels. The network comprises $p_{1} p_{2} \times p^{2}$ transmitters, $p_{1} p_{2} \times p^{2}$ receivers, and $p^{2}+p$ star couplers. Each user is assigned a single wavelength/time/spatial matrix codeword.

The 3D OCDMA system encoder is shown in Figure 2. An electrooptical modulator $(\mathrm{EOM})$ is realized when the information bits for the user $\#(\alpha, \beta, \gamma)$ using on-off shift-keying scheme and the broadband laser source (BLS). The resulting optical signals are encoded with the specific code matrix $\mathbf{C}_{\alpha, \beta, \gamma}$ in two steps: wavelength/time coding, that is, block $\mathbf{A}_{\alpha, \beta}$, and spatial encoding. The wavelength/time encoding structure is implemented by a group of FBGs and tunable optical delay lines. The FBG center wavelength and delay time are determined by the CHP code. The spatial encoding process is realized through connections between the splitter and the star couplers, which are determined by the chip value of MP code. The main function of switch controllers is to connect or unconnect to the couplers according to the MP code patterns in spatial domain of 3D OCDMA codes.

The 3D OCDMA system decoder is shown in Figure 3. The proposed double balanced detector can be divided into two branches, and each branch contains one wavelength/time balance detector. The connection between the two branches and the star couplers agrees with the user's desired spatial codeword and complement, respectively. After passing through combiners, the signal is received by the same wavelength/time decoding process. The attenuators $\delta 1$ and $\delta 2$ shown in Figure 3 are used to eliminate multiuser interference in the frequency and spatial domains, respectively [9]. Because of the effects of various noises in the PDs, the decision variable is a random variable, and a decision device is used to determine whether the transmitted signal is logical "1" or logical " 0 ."

\section{Performance Analysis of 3D OCDMA Network}

To analyze the error probability of the 3D OCDMA system in this study, we ignore the thermal and shot noises in the photodetection process and only consider the interference from other users. The exact probability of error per bit $P_{e}$ is defined as

$$
\begin{aligned}
P_{e}= & P_{r}\left(Z_{1} \geq \frac{\mathrm{Th}}{b_{k}}=0\right) \cdot P_{r}\left(b_{k}=0\right) \\
& +P_{r}\left(Z_{1} \geq \frac{\mathrm{Th}}{b_{k}}=1\right) \cdot P_{r}\left(b_{k}=1\right),
\end{aligned}
$$




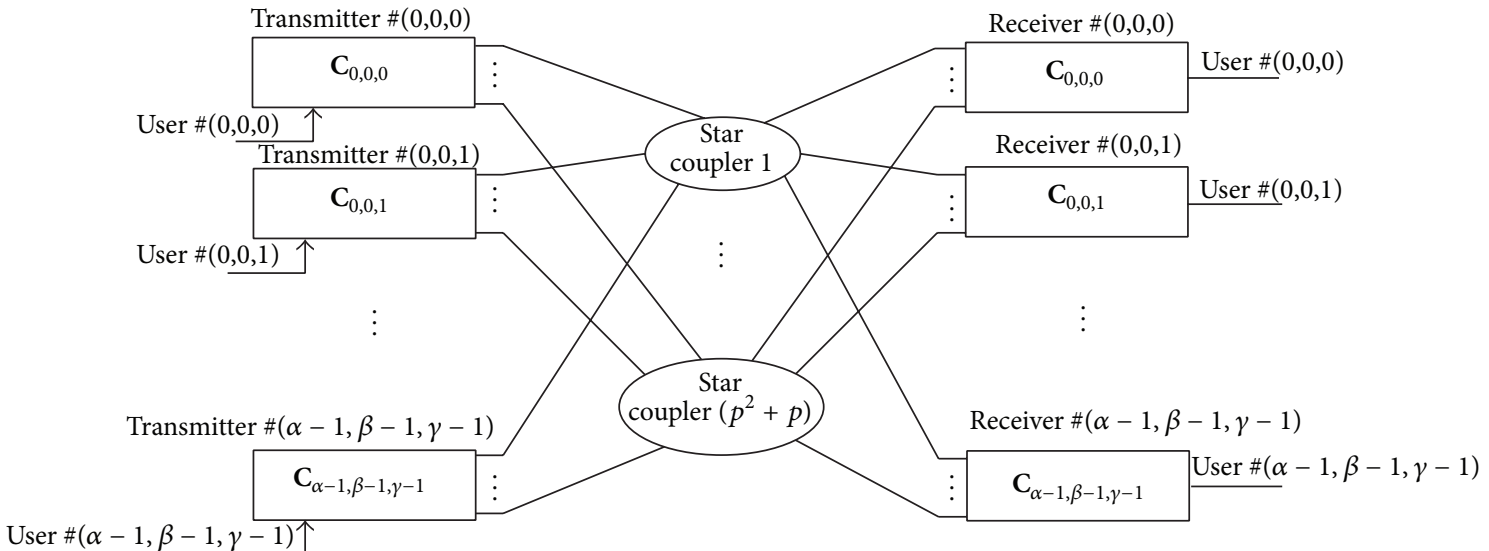

FIGURE 1: Conceptual schematic block diagram of the wavelength/time/spatial OCDMA network.

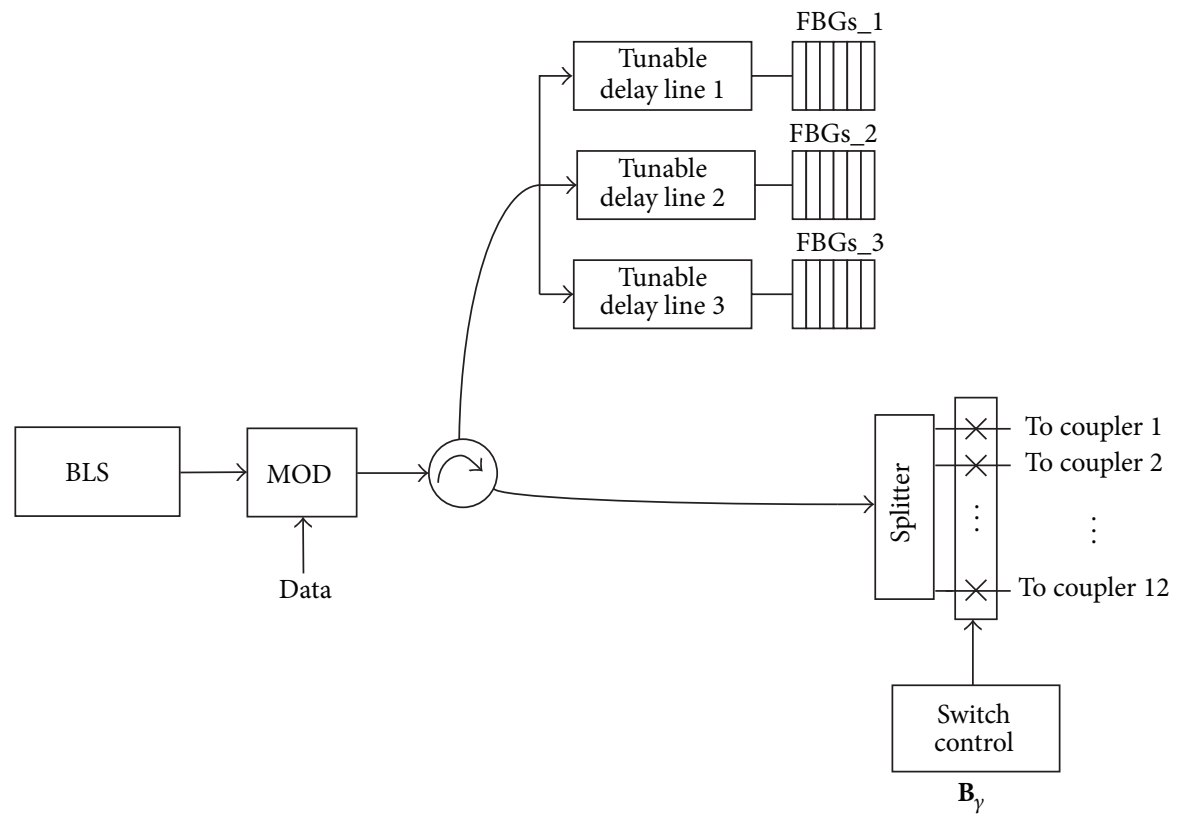

Figure 2: Encoder for CHP/MP code $\left(p_{1}=p_{2}=t=p=3\right)$.

where $Z_{1}$ is the current at the output of the double balanced detector, $b_{k}$ is the data bit of the $k$ th user, and Th is defined as the threshold. The second term of $(8)$ is zero for $0 \leqq T h \leqq K$ [2], where $K$ is the total number of simultaneous users in the system, and the first term of (8) can be written as

$$
P_{e}=\int_{\mathrm{Th}}^{\infty} P_{I}(I) d I
$$

where $I$ is the interference signal and $P_{I}(I)$ is the probability density function for the interference signal $I$. For mathematical convenience, we assume that all chips are synchronized. Under this assumption, (9) can be expressed as

$$
P_{e}=\frac{1}{2} \sum_{i=\mathrm{Th}}^{K-1} C_{i}^{K-1} q^{i}(1-q)^{K-1-i},
$$

where $q$ is the cross correlation probability (we explain how to calculate $q$ in the next paragraph).
To analyze the performance of 3D OCDMA system with $\left(t \times p_{1} p_{2} \times\left(p^{2}+p\right), w, \lambda_{a}, \lambda_{c}\right) \mathrm{CHP} / \mathrm{MP}$ codes, we must calculate the probability $q$. With this property, we can obtain a weight of 1 for the synchronous cross correlation between two distinct codewords during the detection process. Because the $2 \mathrm{D} \mathrm{CHP}$ code has one pulse per row, we calculate $q_{1}=t /(2 \times$ $p_{1} p_{2}$ ) [8]. For the $3 \mathrm{D} \mathrm{CHP} / \mathrm{MP}$ code, the cross correlation probability can be expressed as

$$
q_{2}=\frac{p^{2}-1}{p^{2}} q_{1}+\frac{1}{p^{2}} C_{1}^{p+1} q_{1},
$$

where $p^{2}$ is the number of MP codes used for spatial coding.

The first term of (11) is the probability that two distinct $\mathrm{CHP} / \mathrm{MP}$ codes have different spatial coding schemes, and the second term is the probability of both codes having the same spatial coding scheme. We replace $q_{2}$ of (11) with $q$ to substitute (10) and let $\mathrm{Th}=w$. 


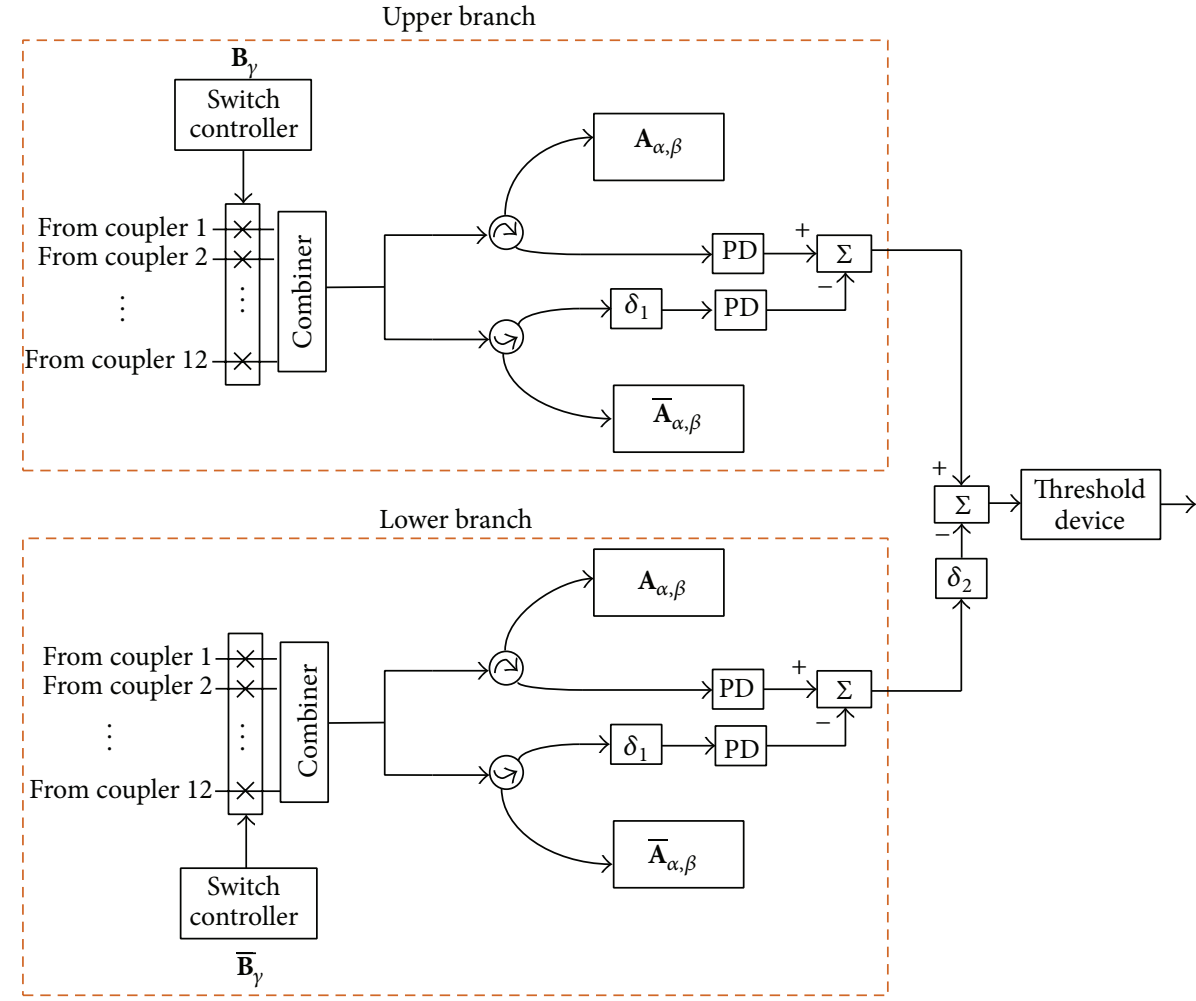

FIgURE 3: Decoder for the corresponding CHP/MP code.

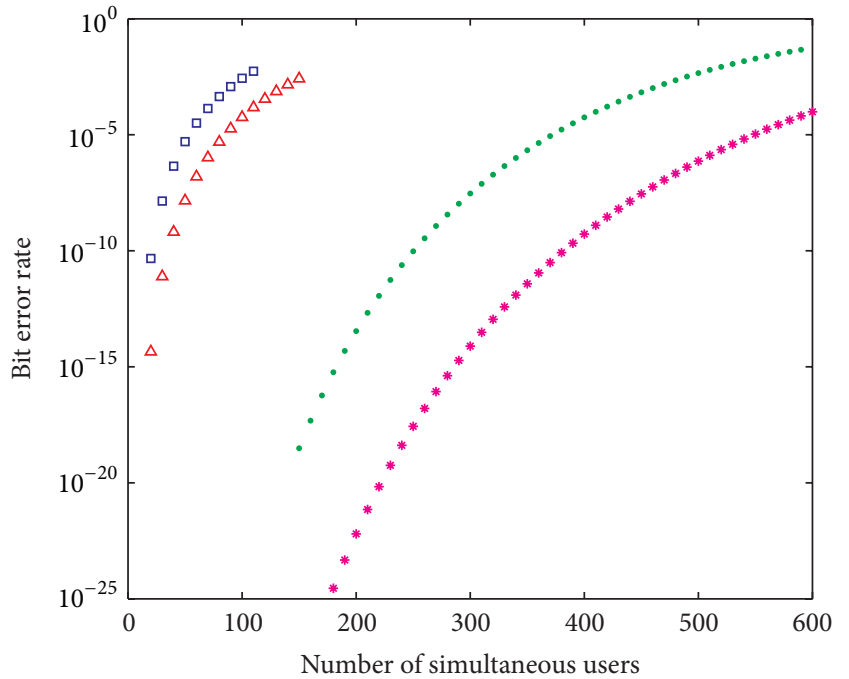

- CHP code for $p_{1}=p_{2}=t=11$

$\triangle$ CHP code for $p_{1}=p_{2}=t=13$

- Hybrid CHP/MP code for $p_{1}=p_{2}=t=11, p=3$

* Hybrid CHP/MP code for $p_{1}=p_{2}=t=13, p=3$

FIgURE 4: BER versus number of simultaneous users.

Figure 4 shows the variation of $\mathrm{BER}$ according to the number of simultaneous users. The CHP/MP code cardinality increased significantly compared to the CHP code under the same BER, as shown in Figure 4. The results in Figure 4 indicate that system performance can be enhanced using the 3D code method because the beating terms and multipleaccess interference (MAI) were both reduced by the double balanced detection mechanism. Additionally, the optical component can be relaxed for high transmission scenery.

\section{Conclusion}

This study proposed a new 3D CHP/MP code with a double balanced detector for OCDMA networks. Although synchronization between fiber channels must be considered during the decoding process, the advantage of cardinality is increased significantly compared to that of traditional OCDMA networks. The codes construction is based on prime codes' family; the 3D codes generation is straightforward. Additionally, beat noise and multiuser interference are suppressed efficiently by the double balanced detection mechanism proposed in this study. A comparison of designed $3 \mathrm{D}$ method and traditional 2D CHP coding scheme shows 3D system with spatial coding domain can support almost more than two hundred simultaneous users at the same bit error rate scenario. According to the reason, the new $3 \mathrm{D}$ design could be useful for the development of future OCDMA networks.

\section{Conflict of Interests}

The authors declare that there is no conflict of interests regarding the publication of this paper. 


\section{Acknowledgment}

This study was partially supported by the National Science Council under Grant no. 102-2622-E-150-016-CC3.

\section{References}

[1] J. A. Salehi, "Code division multiple-access techniques in optical fiber networks Part I. Fundamental principles," IEEE Transactions on Communications, vol. 37, no. 8, pp. 824-833, 1989.

[2] J. A. Salehi and C. A. Brackett, "Code division multipleaccess techniques in optical fiber networks Part II. Systems performance analysis," IEEE Transactions on Communications, vol. 37, no. 8, pp. 834-842, 1989.

[3] J. A. Salehi, A. M. Weiner, and J. P. Heritage, "Coherent ultrashort light pulse code-division multiple access communication systems," Journal of Lightwave Technology, vol. 8, no. 3, pp. 478491, 1990.

[4] H. Heo, S.-S. Min, Y. H. Won, Y. Yeon, B. K. Kim, and B. W. Kim, "A new family of 2-D wave-length-time spreading code for optical code-division multiple-access system with balanced detection," IEEE Photonics Technology Letters, vol. 16, no. 9, pp. 2189-2191, 2004.

[5] C. C. Yang and J. F. Huang, "Two-dimensional M-matrices coding in spatial/frequency optical CDMA networks," IEEE Photonics Technology Letters, vol. 15, no. 1, pp. 168-170, 2003.

[6] S. Kim, K. Yu, and N. Park, "New family of space/wavelength/ time spread three-dimensional optical code for OCDMA networks," Journal of Lightwave Technology, vol. 18, no. 4, pp. 502$511,2000$.

[7] B.-C. Yeh, C.-H. Lin, and J. S. Wu, "Noncoherent spectral/time/spatial optical CDMA system using 3-D perfect difference codes," Journal of Lightwave Technology, vol. 27, no. 6, pp. 744-759, 2009.

[8] T. H. Shake, "Security performance of optical CDMA against eavesdropping," Journal of Lightwave Technology, vol. 23, no. 2, pp. 655-670, 2005.

[9] G.-C. Yang and W. C. Kwong, "Performance comparison of multiwavelength CDMA and WDMA + CDMA for fiber-optic networks," IEEE Transactions on Communications, vol. 45, no. 11, pp. 1426-1434, 1997. 


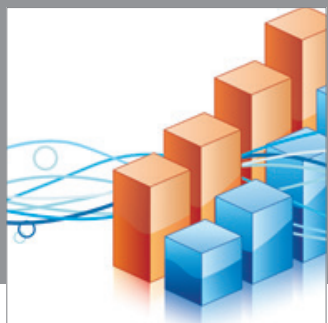

Advances in

Operations Research

mansans

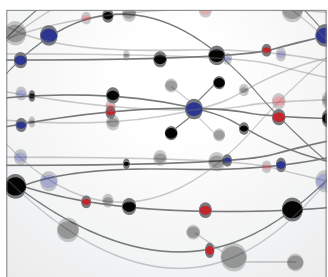

The Scientific World Journal
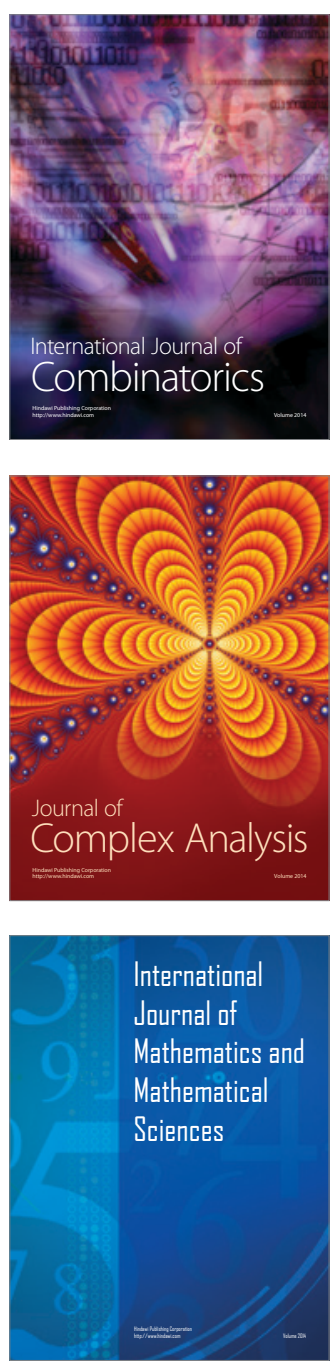
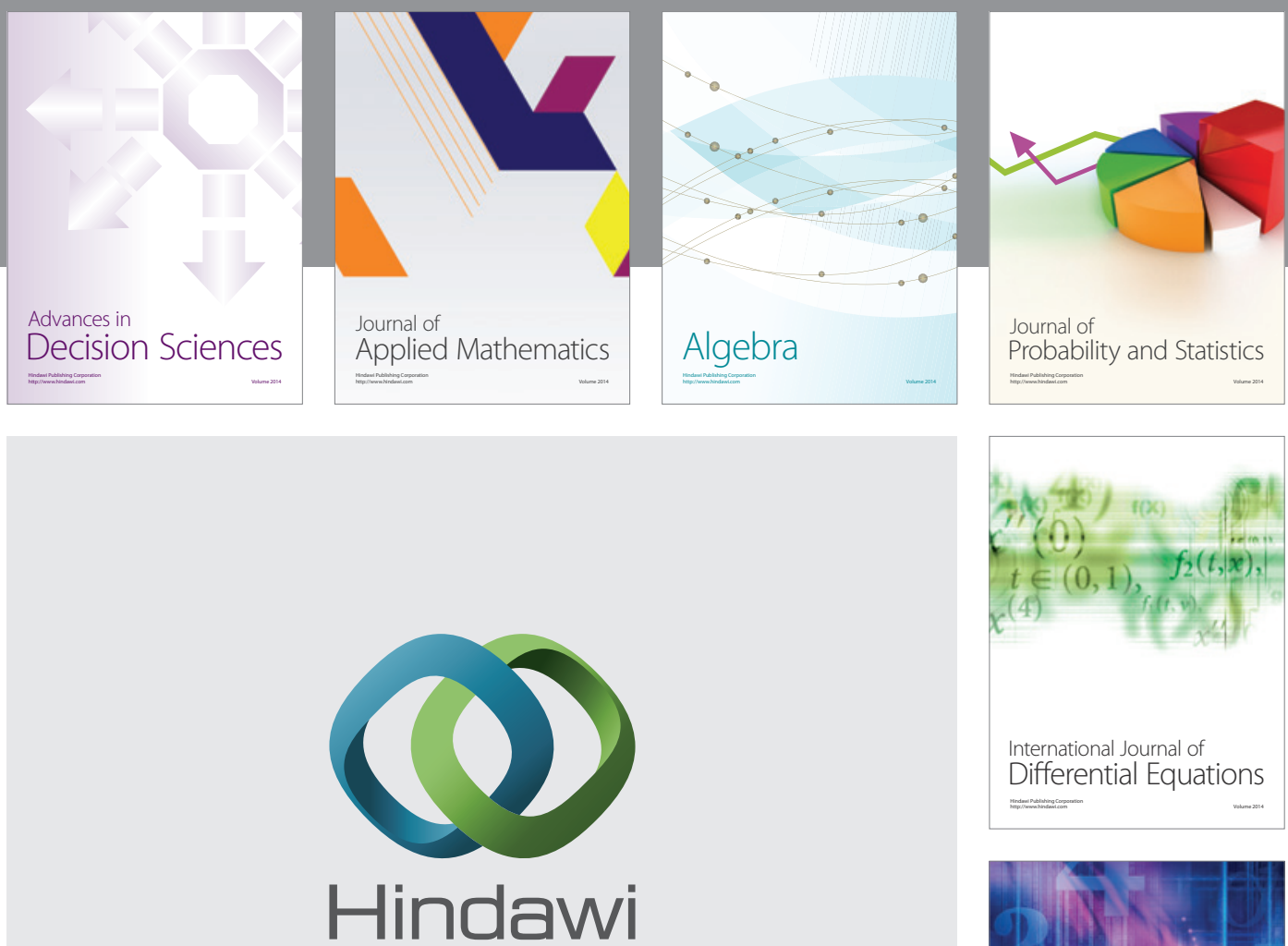

Submit your manuscripts at http://www.hindawi.com
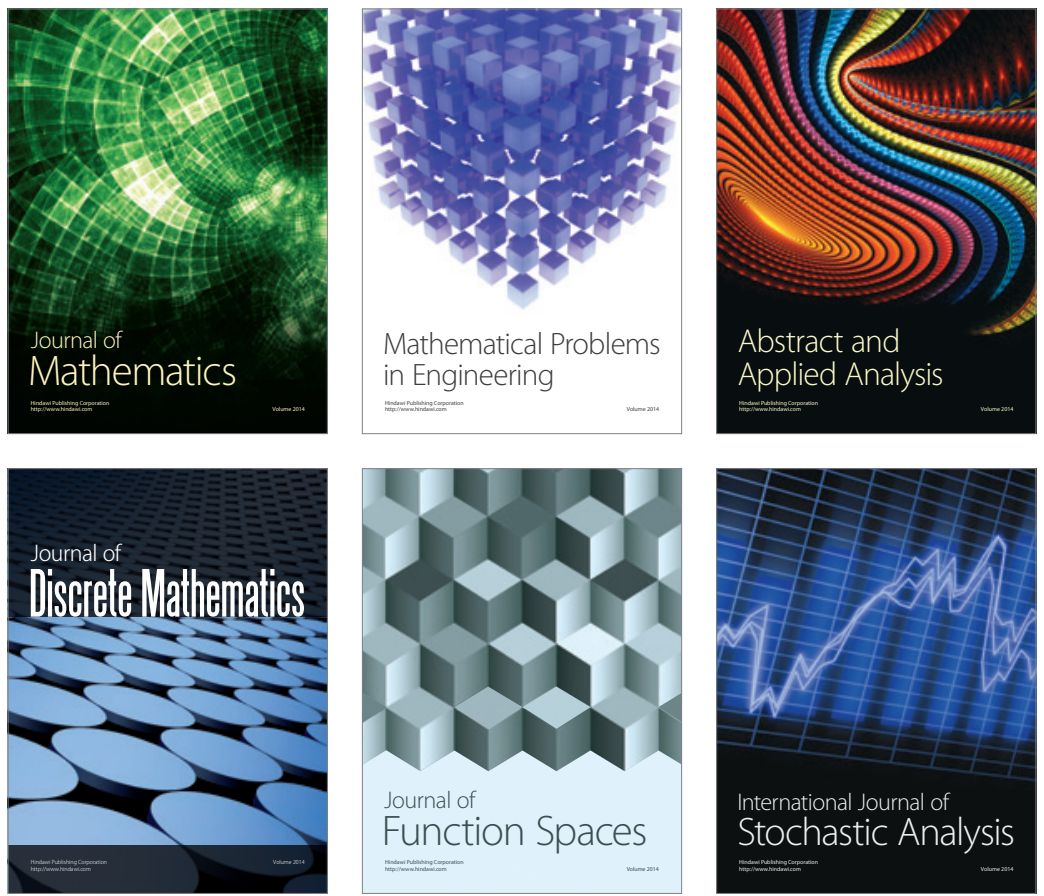

Journal of

Function Spaces

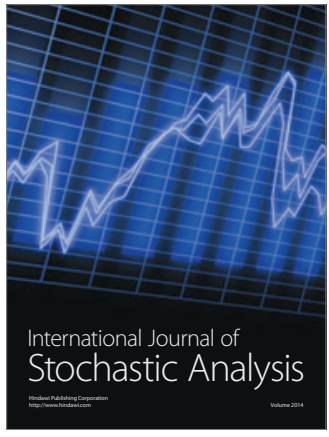

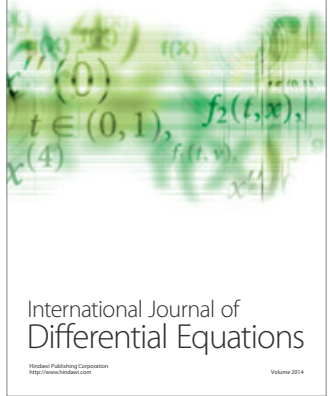
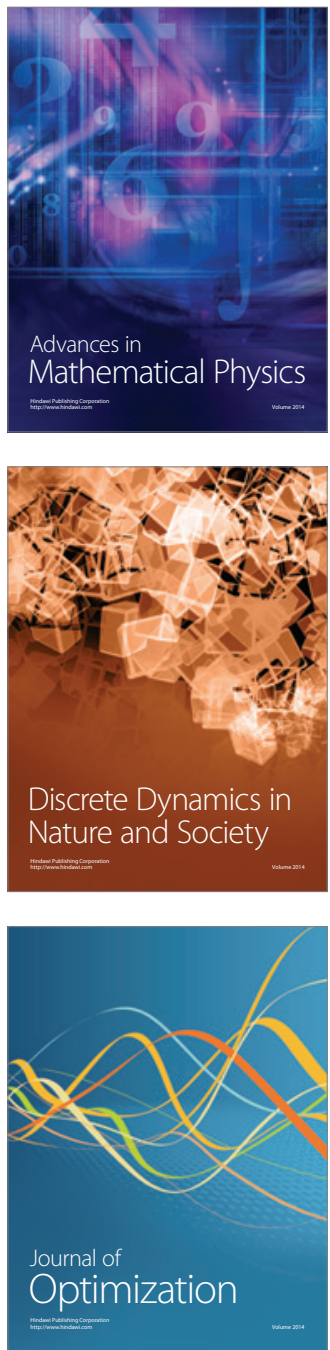